\title{
Traditional Eating Habits and Level of Homocysteine in the Acute First-Ever Ischaemic Stroke
}

\author{
Bajram Kamberi ${ }^{1}$, Gojart Kamberi² \\ ${ }^{1}$ Clinical Hospital Tetovo, Tetovo, Republic of Macedonia; ${ }^{2}$ State University of Tetovo, Tetovo, Republic of Macedonia
}

\begin{abstract}
Citation: KamberiB, KamberiG. Traditional Eating Habits and Level of Homocysteine in the Acute First-Ever Ischaemic Stroke. Maced J Med Sci. 2012 Jul 31; 5(2):181-188, http///dx doi org/ 10.3889/MJMS.1957-5773.2012.0231.

Key words: dietary habits; hyperhomocisteinemia; meal with high-fat content; acute first-ever ischaemic stroke; Republic of Macedonia.

Correspondence: Bajram Kamberi, Prim.Mr.Sci. Clinical Hospital Tetovo, Tetovo 1201, Republic of Macedonia Phone: 072-239-014 e-mail: bajram_kamberi@yahoo.com

Received: 02-Mar-2012; Revised: 20-Mar-2012; Accepted: 28-Apr-2012; Online first: 29-Jun-2012

Copyright: $\odot 2012$ Kamberi B. This is an open access article distributed under the terms of the Creative Commons Attribution License, which permits unrestricted use, distribution, and reproduction in any medium, provided the origin author and source are credited.

Competing Interests: The authors have declared that no competing interests exist.

Background: Meal with high-fat content represents a common and increasing habit in general population in the Macedonia. Contribution of homocysteine and meal with high-fat content in the acute phase of first-ever ischaemic stroke (AFIS) due to the different dietary habits remains unclear.

Aim: Our aims/hypothesis was comparison of serum total homocysteine (tHcy) level and dietary habits in patients with AFIS and in controls due to non-consuming (non-users) or consuming (users) meat with high content of fats that can be regarded as traditional eating habits influenced by religion dietary restrictions from Lower Pollog populations.

Materials and Methods: From initially 847 patients hospitalized from September 2008 to August 2010in neurology department, only 231 patients with diagnostic entity-AFIS and 194 healthy subjects were evaluated in this study. Serum tHcy was measured on admission and dietary history was noted.

Results: In the overall pooled estimate, for all included study participants, serum tHcy level in the acute phase of disease and frequency of users are similar in patient with AFIS and in controls, but non-users mostly was dispose to AFIS and hyperhomocysteinemia.

Conclusion: Meat with high-fat content is very often combined with tHcy-increasing effects at users. Because the regression coefficient of linear regression model with users as variable, adjusting for age, gender and serum tHcy of patients with AFIS was not confirmed the statistical significance of previous pretesting factors by cross tabulation model our research could not confirm that hyperhomocisteinemia and meat with high-fat content are the only reason for cause of AFIS.
\end{abstract}

\section{Introduction}

Cardiovascular diseases (CVD) are the main cause of death and a major cause of hospitalization in European countries [1], including the Republic of Macedonia [2]. During the last decades, epidemiological data showed wide differences of mortality rates for CVD within these countries $[1,2]$. These vast differences have been mainly attributed to environmental factors and in particular to differences in dietary pattern [1, 2], especially in favour of Mediterranean countries where low rates of cardiovascular-related mortality have been observed [3]. The appearance of acute first-ever ischemic stroke (AFIS) [4] is usually sudden and is aroused by a number of traditional and newer risk factors some of which are non modifiable and some are modifiable [5].

Moderately elevated of all circulating plasma total homocysteine (tHcy) concentrations in the blood (hyperhomocysteinemia) [6] and intake meal with highfat content $[7,8]$ as increasing dietary habits [9], both are cardiovascular risk factors highly influenced by diet [3], 
that may be determined endothelial vascular dysfunction [10-12], as well development of atherosclerosis for decades $[13,14]$ until accelerated and the onset of its first clinical manifestations [15-19].

However, despite a worldwide promotion of Mediterranean diet as a model for healthy eating $[1,3$, 20,21] and observed difference in diet concerned healthy lifestyle and vascular risk factors, in particular of homocysteine-decreasing effects [22], as well traditional eating habits followed by the people of the Mediterranean region [23], a progressive shift to Westernized food habits has developed in southern European regions [24, 25] also in Republic of Macedonia [26], whereas the original cultures concerning nutrition are about to be lost [25].

On the contrary, populations that maintained their traditional food habits have been shown to have a decreased risk of mortality [24]. Some authors recognized that relevant differences in dietary pattern has been strongly reflected by an increase in the prevalence of CVD, including stroke and those were highly influenced by religion dietary restrictions characterized by consuming primarily meats with high content of fats, in particularly pork meat, lard, bacon and meat products, as well as the preference for consumption of whole milk and dairy products made of it $[2,27,28]$. This habitual consumption from the same authors especially in relation to risk factor for cerebral infarction via its atherogenecity [21] was evaluate as variant of traditional dietary fat habits and to provide a significant model for inadequate eating [2, 27] or badness dietary habits [29]. In Lower Pollog, a high hospitalization rate of patients started three decades ago [29] with cerebral infarction becoming the most frequent stroke subtype [29, 30] contributes to excess different neurological diseases [31], but data about nutritional habits from this area still not refer.

Thus, the aims/hypothesis of this study was evaluating the influence of different dietary fat habits (referred to hereafter as simply "user" versus "nonuser") on tHcy concentrations to our study cohort; ethnically diverse population with unique dietary habits: traditionally, pork is eaten only by Christians and not by Muslims [23].

\section{Materials and Method}

\section{Recruitment, inclusion and exclusion criteria}

This research designed to assess the potential effects of traditionally dietary habits (converted as users and non-users) on fasting levels of tHcy and their influence on the incident AFIS was an ancillary of previous experience study who conducted for a period of two years. Both patients and controls were selected from cohort of 1050 participants who were similar with respect to geographical location (Lower Pollog is included in stratum of the region that death-rate from cardiovascular diseases is to 399 in 100.000 citizen [2] and doctrine status: Orthodoxy Christian and Mohammedan origin as predominant religion are reflex the habitual diet of Macedonian, Albanian, Turkish, and Serbian and Romany participants [29].

The study neurologist arranged an interview uring the patient was treatment in the hospital. The secondary author approached control subjects at random and recruited and interviewed them while they were waiting for pay a visit at inpatient. All participants were assured of confidentiality and were advised of their right to withdraw from the project at any time without prejudice before giving formal consent. Each interview took an average of 5 minutes to complete.

Detailed descriptions of the design and methods and of its recruitment procedures and main results have been published [31]. In brief, initially the group 1 (examined group) consisted of 847 consecutive patients with different neurological disease hospitalized in our department, a main referral department which serves an area with 200.000 inhabitants in northwest Macedonia and the group 2 (control group) consisted of 203 healthy volunteers from community-based inhabitants free of neurological diseases, following the same exclusion criteria as applied for cases.

At recruitment, diagnosis of ischemic stroke was determined by a neurologist, based on the results of strict neurological examination (evaluation of the existing sudden onset of a new focal neurological event with a lesion of vascular origin and symptoms that persisted for more than 24 hours, subsequent confirmation of infarction in the brain by computed tomography (CT) or cranial magnetic resonance imaging scans (MRI). Cerebral CT scans or MRI reports were performed by qualified radiologist in all cases. Therefore, we considered only patients with a first-ever ischemic stroke (thrombotic or embolic) defined according to the literature [4]. After excluding the subjects as per the exclusion criteria like transient ischemic attack, primary subarachnoid or cerebral hemorrhage, with history of cerebrovascular disease or an organic psycho syndrome, drug treatments which influence homocysteine level (use of 
anticonvulsants, multivitamins, etc.), and whose homocysteine concentrations not were determined, a total number of 231 newly diagnosed ischaemic stroke patients, according to the complete medical history admitted between September 1, 2008 and August 31, 2010 to the Neurology Department of Clinical Hospital Tetovo, and 194 clinically healthy subjects of Lower Pollog were included into the study.

We also excluded at baseline, all participants who refused to give written informed consent to participation in the study, underwent completed the anamnesis with a detailed somatic neurological examination or donated a fasting blood sample for biochemical measurements. The study protocol was reviewed and approved by Board of our hospital.

The occurrence of a risk factor was determined from results of clinical examination, diagnostic procedures, or evidence in the medical records. The definition of each risk factor follows. Subjects were considered to have hyperhomocysteinemia if they had a serum tHcy concentration $\geq 15 \mathrm{mmol} / \mathrm{L}$ [6].

The independent effects of two specific traditional dietary fat intake (referred to hereafter as simply "user" versus "non-user") differ in terms of environmental factors [23] were estimated based on the response to the a diet questionnaire designed for the purposes of this study. The data was completed with personal and demographic information on each subject such as birth date, birthplace, nationality, religion and medical history. Depending on the respondent's language preference, the foodfrequency data were prospectively collected as part of the interviewed face-to-face in Macedonian or in Albanian language by study neurologist during the anamnesis. When patients were unable to provide answers because of the effects of the AFIS, the answers using such proxy informa-tion were obtained from their next of kin. The questionnaire consist one question about traditional eating habits in their families and to whom they will first refer, including the consumption frequency of red meat from pork ("during the past 30 days, how often did you eat meat pork based on four categories: never, once/ week, once/month, and almost daily"). Their answers could not be reliably quantified, but, were analyzed with respect to tHcy distribution by frequency of consumption stratified in four habitués group: almost daily (scored as always), once/week (scored as frequently), and once/ month (scored as sometimes), whereas those who did not consume pork meat represented the reference group (scored as never). All participants were categorized into users and non-users according to meat intake distribution influenced by religion dietary restrictions. The effects of both non-consumption and pork meat consumption only (never/users) and frenzy pork meat consumption solely (never/always, never/frequently, never/sometimes) were calculated.

\section{Homocystiene assay}

In all participants including our study group, blood samples were collected by venipucture in the department according to standard protocol in the morning (8.00-9.00) after a 12-hour overnight fast in tubes without anticoagulant immediately put in ice (for homocysteine assay) and transferred to our biochemical laboratory, where centrifuged within 15 min (3000 cycle for 10 min at room temperature). After priority analysis, surplus sera were frozen at $-18^{\circ} \mathrm{C}$ until assay. Serum levels of tHcy were determined by enzymatic assay [13] on an automatic analyzer (Hitachi 902) using commercial kits [Diazyme laboratories, Germany) and standard laboratory procedures as previously described [14]. The reference intervals were defined according to the guide of manufactured and according to the literature [6].

\section{Statistical analysis}

Statistical analysis was done using SPSS version 17.0. We used descriptive statistics to characterize participants. After comparing the traditional eating diet of case patients and control subjects, we conducted several statistical methods have been used for the statistical analysis of the data obtained in the course of the study: the measures of central tendency; the structure percentages; independent sample test (t-test for independent samples, Chi-Square test) and correlation test (Spearman's rho Ordinal by Ordinal Correlation). To assessment of exposure we reported either odds-ratios (ORs; case-control subjects) across several different categories of users/non-users across case with AFIS and control subjects. To confirm the statistical significance between possibility of a relationship between meat intake distribution influenced by religion dietary restrictions (as the dependent variable) and AFIS risk, adjusting for confounding variables (ie age, gender and serum tHcy) of patients with AFIS was used multivariate linear regressive analysis (including all group consisted of 231 patients), using the "Backward" model and results were expressed as regression coefficient (b) standard error (s.e.). A $p$ value of $p<0.05$ was considered to indicate statistical significance. 


\section{Results}

Of the 435 who started run-in, $425(97.70 \%)$ randomized participants (225 male, 200 female) had serum thcy values measured and were included in the statistical analysis. Baseline characteristics of the patients and controls are show in Table 1.

Table 1: Age of the examinees.

\begin{tabular}{lcccccc}
\hline Age & $\mathrm{N}$ & Mean & Min. & Max. & Std.Dev. & Std.Err. \\
\hline Examined group & 231 & 65.70 & 37 & 87 & 9.756 & 0.642 \\
Control group & 194 & 52.09 & 18 & 85 & 14.486 & 1.040 \\
\hline
\end{tabular}

T-test for independent samples $=11.135 ; \mathrm{p}<0.001$.

There were 231 patients with ischemic stroke, whose mean age was 65.70 (37-87) years; among them,105 were females. Controls were at significantly younger average age than the examined group $(p=0.000)$. With respect to the doctrine, there was no statistically

Table 2: Baseline characteristics of participants by religion group.

\begin{tabular}{lcccc}
\hline Variable & Chrystians & Muslims & test & $\mathrm{P}$ \\
\hline $\mathrm{n}$ & 122 & 303 & & \\
Male, \% (n) & $50.82(62)$ & $53.79(163)$ & $\mathrm{t}=0.555$ & 0.579 \\
Age (years) & $60.38 \pm 11.71$ & $59.13 \pm 14.69$ & $\mathrm{t}=0.919$ & 0.359 \\
Fasting tH cy (mmol/L) & $16.89 \pm 6.61$ & $17.61 \pm 6.669$ & $\mathrm{t}=1.005$ & 0.316 \\
Hyperhomocysteinemic, \% (n) & $51.64(63)$ & $59.07(179)$ & $\mathrm{x}^{2}=1.962$ & 0.161 \\
Never consumer*\% $\%$ (n) & $7.38(9)$ & $100(303)$ & $\mathrm{x}^{2}=382.292$ & $\mathrm{p}<0.001$ \\
\hline
\end{tabular}

*of red meat from pork.

significant difference between recruit 122 Christians and 303 Muslims regarding gender, age, fasting tHcy and percent of hyperhomocysteinemic subjects; never consumer was found to be the only statistically significant risk factor in all study groups. Comparisons of these characteristics in our sample are revealed in Table 2.

As expected, Muslims never consume pork meat (100\%). Also, there was evidence of an increased risk of incident AFIS linked to religion distribution in our cohort study (ORs $=1.278,95 \% \mathrm{Cl}=0.839-1.948)$, notably to Muslims patients (ORs $=1.073,95 \% \mathrm{Cl}=$ $0.950-1.214$ ), but at Christians is missing (ORs $=0.840$, $95 \% \mathrm{Cl}=0.623-1.133)$. Thereof reason, change in fasting levels of thcy adjusted for meat intake distribution influenced by religion dietary restrictions (converted as users and non-users) was the primary outcome variable in the examined group compared with control. Hence, after their first examination according to its self reported common diet as part of traditional eating habits, 231 randomly selected patients were categorized to participate into two subgroups: 56 users and 175 nonusers, also 194 control subjects, into 57 users and 137 non-users. Demographic and behavioral characteristics of subjects enrolled in the study are summarized in Table 3.

As show in the table above, the examined and control subgroups were statistically equalized for frequency of users. Although controls $(p=0.040)$ were younger than patient subgroups $(p=0.446)$, tHcy levels did not differ according to gender between the groups ( $p$ $>0.05$ ).

Those who did not consume pork meat were more common in the patients $(75.76 \%)$ than in the control group (70.62\%) but mean value of tHcy in users vs. non-users were not significant between patients and

Table 3: Characteristics of the study population and its eating habits, stratified by consumption meat (pork) and level of homocysteine.

\begin{tabular}{|c|c|c|c|c|c|c|}
\hline & \multicolumn{2}{|c|}{ Examined group } & \multicolumn{4}{|c|}{ Control group } \\
\hline & $\begin{array}{c}\text { Non-users } \\
(n=175)\end{array}$ & $\begin{array}{l}\text { Users } \\
(n=56)\end{array}$ & $\begin{array}{l}\text { Statistical } \\
\text { analysis }\end{array}$ & $\begin{array}{c}\text { Non-users } \\
(n=137)\end{array}$ & $\begin{array}{l}\text { Users } \\
(n=57)\end{array}$ & $\begin{array}{l}\text { Statistical } \\
\text { analysis* }\end{array}$ \\
\hline Age & $65.43 \pm 9.851$ & $66.55 \pm 9.491$ & 0.446 & $50.90 \pm 15.630$ & $54.96 \pm 10.864$ & 0.040 \\
\hline \multicolumn{7}{|l|}{ Gender } \\
\hline Men $(n)$ & $21.59 \pm 6.735(96)$ & $22.57 \pm 4.591(30)$ & 0.372 & $12.99 \pm 2.058(73)$ & $13.38 \pm 2.994(26)$ & 0.535 \\
\hline Women (n) & $20.96 \pm 6.071(79)$ & $22.81 \pm 5.185(26)$ & 0.138 & $11.89 \pm 2.040(64)$ & $10.97 \pm 2.469(31)$ & 0.077 \\
\hline$\%$ (male/female) & $54.86 / 45.14$ & $53.57 / 46.43$ & & $53.28 / 46.71$ & $45.61 / 54.39$ & \\
\hline \multicolumn{7}{|c|}{ Meat (pork) consumption ( $\mathrm{n}, \%)$} \\
\hline Never & $21.31 \pm 6.433(175)$ & $22.68 \pm 4.832(56)$ & 0.093 & $12.47 \pm 2.115(137)$ & $12.07 \pm 2.957(57)$ & 0.351 \\
\hline Per day & 0 & $27.33 \pm 4.812(12)$ & 0.001 & 0 & $14.69 \pm 2.869(16)$ & 0.008 \\
\hline Per week & 0 & $21.94 \pm 4.215(36)$ & 0.459 & 0 & $11.09 \pm 2.414(32)$ & 0.005 \\
\hline Per month & 0 & $19.00 \pm 1.690(8)$ & 0.007 & 0 & $10.89 \pm 2.028(9)$ & 0.049 \\
\hline \multicolumn{7}{|c|}{ Serum tHcy concentration $(n, \%)$} \\
\hline$<15 \mathrm{mmol} / \mathrm{L}$ & $12.18 \pm 1.328(11)$ & 0 & & $11.93 \pm 1.423(122)$ & $11.20 \pm 1.702(50)$ & 0.009 \\
\hline $15-30 \mu \mathrm{mol} / \mathrm{L}$ & $20.351 \pm 3.51(148)$ & $21.388 \pm 3.558(49)$ & 0.081 & $16.867 \pm 1.642(15)$ & $18.286 \pm 2.563(7)$ & 0.215 \\
\hline $30-100 \mathrm{mmol} / \mathrm{L}$ & $36.44 \pm 6.387(16)$ & $31.71 \pm 1.976(7)$ & 0.014 & 0 & 0 & \\
\hline
\end{tabular}

*t-test for independent samples. 
control subgroups $(p>0.05)$.

However, when homocysteine profile of patients was analyzed according to frenzy of pork consumption that unit (the reference group, scored as never: $21.31 \pm$ $6.433 \mathrm{mmol} / \mathrm{L} ; 1 \mathrm{x} /$ day, scored as daily: $27.33 \pm 4.812$ $\mathrm{mmol} / \mathrm{L} ; 1 \mathrm{x} /$ week, scored as weekly: $21.94 \pm 4.215$ $\mathrm{mmol} / \mathrm{L} ; 1 \mathrm{x} / \mathrm{month}$, scored as monthly: $19.00 \pm 1.690$ $\mathrm{mmol} / \mathrm{L}$ ), it was found that mean value of serum tHcy levels was not significantly different between never and to those consuming less than $1 x /$ week $(p=0.459)$, but differences were significant when compared non users to those consuming every day $(p=0.001)$ as well as $1 x /$ month $(p=0.007)$. Beside patients, among the control habitués group, all differences on tHcy distribution were statistically significant. The percentage of subjects with hyperhomocysteinemia was higher at examinee group (95.24\%) than at the control group (11.34\%). These differences were no statistically significant between the users vs. non-users in subjects with moderate hyperhomocysteinemiain the patients (0.081) and control subgroups (0.215). In our group of patients mild hyperhomocysteinemia was found statistically more often in patients those who did not consume pork meat (0.014).

We observed in the overall pooled estimate, for all included study participants, that users mostly was not dispose to AFIS (ORs $=0.825,95 \% \mathrm{CI}=0.602-1.131$ ) after analyzed by cross tabulation model, to assessment of exposure on incident AFIS. Conversely, more exposed was male gender (ORs $=1.069,95 \% \mathrm{Cl}=0.892-1.281$ ), and Muslim subjects (ORs $=1.073,95 \% \mathrm{Cl}=0.950$ 1.214). There was also a significantly increased risk toward AFIS (ORs = 8.398, 95\% Cl = 5.660-12.460) correlated to the hyperhomocysteinemia (Spearman Correlation $=-0.844, p=0.000 ; 95 \% \mathrm{Cl}=0$ ).

With intercession of multivariate linear regressive analysis (including all group consisted of 231 patients), using the "Backward" model, was proved that possibility of a relationship between meat intake distribution influenced by religion dietary restrictions (as the dependent variable) and factors such as age, gender

Table 4: Regression Coefficient of linear regression model between dietary fat intake (pork consumption converted to users/nonusers), age, gender and tHcy levels at patients.

\begin{tabular}{|l|c|c|c|c|c|c|c|}
\hline \multirow{2}{*}{ Model 1 1 } & \multicolumn{2}{|c|}{$\begin{array}{c}\text { Unstandardized } \\
\text { Coefficients }\end{array}$} & $\begin{array}{c}\text { Standardized } \\
\text { Coefficients }\end{array}$ & \multirow{2}{*}{$\mathrm{t}$} & \multirow{2}{*}{ Sig. } & \multicolumn{2}{|c|}{$\begin{array}{c}9.0 \% \text { Confidence } \\
\text { Interval for B }\end{array}$} \\
\cline { 2 - 4 } \cline { 7 - 8 } & $\mathrm{B}$ & $\begin{array}{c}\text { Std. } \\
\text { Error }\end{array}$ & Beta & & & $\begin{array}{c}\text { Lower } \\
\text { Bound }\end{array}$ & $\begin{array}{c}\text { Upper } \\
\text { Bound }\end{array}$ \\
\hline (Constant) & 9.004 & .223 & & 40.331 & .000 & 8.564 & 9.444 \\
Age & -.001 & .003 & -.031 & -.457 & .648 & -.007 & .005 \\
Gender & -.013 & .057 & -.016 & -.237 & .813 & -.126 & .099 \\
tHcy & -.006 & .005 & -.090 & -1.335 & .183 & -.016 & .003 \\
\hline
\end{tabular}

and serum tHcy does not exist any correlation, and this is described in the model below (Table 4).

Notably the regression coefficient of linear regression model with users as variant of traditional dietary fat intake, adjusting for age, gender and serum tHcy of patients with AFIS not confirm the statisticalsignificance of previous pretesting factors by cross tabulation model in our cohort of patients with acute ischemic stroke and control subjects.

\section{Discussion}

In attempt to account for the complexity of nutrition interactions in foods in relation to risk factors for cardiovascular diseases [24] there was observed changing dietary habits of ethnic groups in Europe and implications for health [25]. These findings suggest that the balance of total diet may be most important in determining its physiologic effects in the distribution and control of multiple vascular risk factors and consequently they may contribute in influence geographic variation to differences in stroke incidence and mortality rates [1].

In the Macedonia the statistical data showed wide differences of mortality rates for CVD within regions [2]. These vast differences have been mainly attributing of westernization of the Macedonia lifestyle during 19722000 and are still rising. Meal with high-fat content represent a common and increasing habit in general population in the Macedonia [26]. At the same time trend of differences in dietary pattern has been strongly reflected by an increase in the prevalence of obesity, especially in the youngest population [26], next of ischemic heart diseases [2], or frequency of hyperlipidaemia, hypertension and cerebrovascular insult at Christian subjects [27]. Even if inside the same population relevant differences in dietary habits exist, consuming primarily meats with high content of fats, in particularly pork meat, and dairy products made of it that has been promoted as a model for inadequate eating [2, 27], especially in favour of Macedonia regions where high rates of cardiovascular-related mortality have been observed [2]. The same author showed statistically significant difference between the patients with myocardial infarct and control group for the change of consuming fat [2], while others have recorded increased frequency of the Christian stroke patients beside Muslim $[27,28]$. In reality, Muslims have dietary restrictions due religion and practicing a religious obligation influence their food choices. This may restrict their choices of lean meat [23]. 
Pollog (Lower and Upper) is an area where living different religion groups with different eating habits where low rates of cardiovascular-related mortality have been observed for example in 2001 [2].

Compared to the ethnic Macedonian population the Lower Pollog belongs to the region with relatively high number of hospitalized Albanians [29] with high proportion of AFIS [30]. Probably, demographic characteristics of habitants from Tetovo and its surroundings are simple factor responsible for that [31].

Management of stroke in Lower Pollog is generally beyond the western standards, but probably this fact couldn't be responsible for the whole difference in hospitalization rates [29-31].

At present, data on the traditional eating habits influenced by religion dietary restrictions and data on tHcy-increasing effects of meal with high-fat content are limited largely to Lower Pollog populations and couldn't be compared due to deficiencies of similar comparative studies in clinically healthy subjects in relation to the patients with AFIS enrolled in the study.

Our results documented absence of the statistically significant difference between the homocysteine mean level in different religion populations (higher in Muslims and lower in Christians). According to the results this difference could be partially responsible for the different morbidity from AFIS in these two populations (much higher in Muslims). The mechanism by which thcy could influence the severity of tissue damage caused by ischemia is not clear. El Kossi and Zakhary [10] suggested that homocysteine could be responsible for free radical generation and this way lead to the injury during acute phase of stroke. Similar conclusion was formed by other authors [11].

Unlike us others authors have shows an inverse association between the risk of hemorrhagic stroke and animal protein consumption, in the U.S. Nurses' Health Study cited from Sauvaget at al., [8], also between dietary fat and ischemic stroke in the Framingham study cited in $[2,9]$.

Many of the studies have shown relationship of homocysteine with stroke $[15,16]$ but some could not find the correlation $[18,19]$. We found that concentration of plasma Hcy did differ between cases and controls in the acute phase, distinctive from Happaniemi E, at al., [17] and our finding was not corroborated with results in a more study cited in [6] which found similar levels in cases and controls.
After controlling for possible exposed confounding parameters (ie gender, religion and tHcy levels distribution), our data showed that male gender, Muslim subjects and those with hyperhomocysteinemia were more exposed toward AFIS. Furthermore, then used to identify the independent associations of univariately significant variables with tHcy testing by multivariate linear regressive analysis we not finder associations between ages, sex and tHcy levels of ischemic stroke patients. Other authors draw similar conclusion in the patients with acute ischaemic stroke [19].

Intercommunion between homocysteine levels and meals with high-fat content for each consecutive meal is in a certain sense as possible mechanism action that could be acceleration of greater endothelial dysfunction and higher oxidative stress [12]. Accordingly among other contributors to cardiovascular risk, contribution of homocysteine and traditional eating habits influenced by religion dietary restrictions in our study participants can be regarded probably in picture of association between homocysteine and ischemic stroke and suggestible from codependent on lifestyle and environmental and vascular risk factors [22] and in view of results of our work, we don't supporting thing that only dietary habits of Christians habitants could be regarded as an indicator of bad dietary habits who increased the risk of ischemic heart diseases [2], hyperlipidaemia, hypertension and cerebrovascular insult [27, 28], because in our consecutive series of adult patients with AFIS were three-quarter of those who did not consume pork meat (9 Christians and 303 Muslims).

A major limitation is the case-control design of the study that cannot support evidence for causality but only state hypotheses for the potential implications of the investigated traditional eating habits influenced by religion dietary restrictions as specific nutritional factors in the relationship between homocysteine and AFIS.

Only justification for this study was heterogeneity in the study population (inclusion of five ethnic groups residing in one setting who were stratified in two major religion groups) will provide variance in dietary patterns which is a great advantage when studying the link between diet and disease.

Serum tHcy level in the acute phase of disease and frequency of users are similar in patient with AFIS and in control subjects, but non-users mostly were dispose to AFIS and hyperhomocysteinemia. Because the regression coefficient of linear regression model with 
users as variable, adjusting for age, gender and serum tHcy of patients with AFIS was not confirmed the statistical significance of previous pretesting factors by cross tabulation model our research could not confirm that hyperhomocisteinemia and traditional eating habits influenced by religion dietary restrictions are the only reason for cause of AFIS. Our data demonstrate that traditional eating habits influenced by religion dietary restrictions of the population living in the Lower Pollog area do mirror the features of dietary habits of general population in the Macedonia, as clearly seen by high contribution from non-consumption pork meat with its

product. Thus, these results highlight the need for better understanding of potential elucidation of the molecular mechanisms triggered by elevated tHcy levels and diet as important modifiable determinants of stroke onset.

\section{References}

1. European Stroke Organisation Executive Committee, ESO Writing Commitee. Guidelines for management of ischaemic stroke and transient ischaemic attack. Cerebrovasc Dis. 2008; 25: 457-507.

2. Kosevska D. Elena. Health education in prevention and reduction of risk factors for the onset of ischemic heart diseases in the Republic of Macedonia. Doctoral dissertation; Skopje: Medical Faculty 2004:277.

3. Sofi F, Vecchio S, Giuliani G, Marcucci R, Gori AM, Fedi S, Casini A, Surrenti C, Abbate R, Gensini GF. Dietary habits, lifestyle and cardiovascular risk factors in a clinically healthy Italian population: the "Florence" diet is not Mediterranean. Eur J Clin Nutrition. 2005;59:584-591.

4. Davenport R, Denis M. Neurological emergencies: Acute stroke. J Neurol Neurosurg Psychiatry. 2000; 68:277-88.

5. Hadjiev DI, Mineva PP, Vukov MI. Multiple modifiable risk factors for first ischemic stroke: a population-based epidemiological study. Eur J Neurol. 2003; 10:577-582.

6. Stanger O, Herrmann W, Pietrzik K, Fowler B, Geisel J, Dierkers J, Weger M. D.A.CH.-Liga Homocystein (German, Austrian and Swiss Homocysteine Society): Consensus Paper on the Rational Use of Homocysteine, Folic Acid and B-vitamins in Cardiovascular and Thrombotic Disease: Guidelines and Recommendations. Clin Chem Lab Med. 2003; 41(11):13921403.

7. Tashuizen ME, Nieuwland R, Scheffer PG, Sturk A, Heine RJ, Diamant M. Two consecutive high-fat meals affect endothelial-dependent vasodilatation, oxidative stress and cellular microparticles in healthy men. $\mathrm{J}$ Thrombosis and Haemostasis. 2006;4:1003-1010.
8. Sauvaget C, Nagano J, Hayashi M, Yamada M. Animal Protein, Animal Fat, and Cholesterol Intakes and Risk of Cerebral Infarction Mortality in the Adult Health Study. Stroke. 2004; 35:1531-1537.

9. Ding EL, Mozaffarian D. Optimal dietary habits for the prevention of stroke. Semin Neurol. 2006;26(1):11-23.

10. El Kossi MMH, Zakhary MM. Oxidative stress in the context of acute cerebrovascular stroke. Stroke. 2000; 31:1889-1892.

11. Austin RC, Lentz SR, Werstuck GH. Role of hyperhomocysteinemia in endothelial dysfunction and atherothrombotic disease. Cell Death Differ. 2004; 11(suppl 1):S56-64.

12. Fischer M, Schaebitz W. An overview of acute stroke, therapy past, present and future. Arch Intern Med. 2006; 160: 3196-3206

13. Dou C, Xia D, Zhang L, Chen X, Flores P, Datta A, Yuan C. Development of a novel enzymatic cycling assay for total homocysteine. Clin Chem. 2005;51:1987-1989.

14. Mirkoska M, Grncharoska M, Berisha S. Determination the total homocystein in patients with diabetes type 2 and their children. Balcan J Clin Laboratory. 2009;17(1):106.

15. Eikelboom JW, Hankey GJ, Anand SS. Association between high homocysteine and ischemic stroke and due to large and small-artery disease. Stroke. 2000; 31: 1069-1075.

16. Matsui T, Arai H, Yuzuriha T. Elevated plasma homocysteine levels and risk of silent brain infarction in elderly people. Stroke. 2011; 32: 1116-1119.

17. Happaniemi E, Helenius J, Soinne L, Syrjala M, Kaste M, Tatlisumak T. Serial measurements of plasma homocysteine levels in early and late phases of ischaemic stroke. Eur $\mathrm{J}$ Neurol. 2007; 14:12-17.

18. Fallon UB, Elwood P, Ben Shloma Y. Homocysteine and ischemic stroke in men. J Epidemiol Comm Health. 2001; 55:91-96.

19. Cheryl DB, Larry BG. Homocysteine testing in patients with acute ischaemic stroke. Neurology. 2002; 59(10):1541-1546.

20. Chrysohoou C, Panagiotakos DB, Pitsavos C, Das UN, Stefanadis C. Adherence to the Mediterranean diet attenuates inflammation and coagulation process in healthy adults: The ATTICA Study. J Am Coll Cardiol. 2004;44:152-158.

21. Simopoulos A. Omega-3 fatty acids and cardiovascular disease: The epidemiological evidence. Environ Health Prev Med. 2002;6:203-209.

22. O‘Callaghan P, Meleady R, Fitzgerald T, Graham I; the European COMAC group. Smoking and plasma homocysteine. Eur Heart J. 2002; 23:1580-1586.

23. Dahl M. Middle Eastern Nutrition. Health Care Food and 
Nutrition Focus. 2004; 21(5):6-8.

24. Louise H Dekker, Marieke B Snijder, Marja H Beukers, Jeanne HM de Vries, Henny AM Brants, Evelien J de Boer, Rob M van Dam, Karien Stronks, Mary Nicolaou. A prospective cohort study of dietary patterns of non-western migrants in the Netherlands in relation to risk factors for cardiovascular diseases: HELIUS-Dietary Patterns. BMC Public Health. 2011; 11: 441.

25. Penelope A Gilbert, Santosh Khokhar. Changing dietary habits of ethnic groups in Europe and implications for health. Nutrition Reviews. 2008; 66 (4):203-215.

26. Ministry of Health. Food and Nutrition. Action Plan of the Republic of Macedonia. Skopje, April 2004:9-10. www.rzzz.org.mk.

27. Ejub G, Jankovska S, Angjeleska L, Arbutina-Gruevska S, Pejkovska S. Vlijanie na navikite i obichaite vo ishranata vrz pojavata na hiperlipidemijata, hipertenzijata i mozochnite insulti. Maked Med Pregled. 2004; suppl.61:33.
28. Petrovic-Oggiano G, Damjanov V, Vucic V, Gurinovic M, Popovic T, Debeljak Martasic J, Nikolic M, Avramovic N, Glibetic $M$. Nutrition based on different origin of fats as a risk factor for cerebrovascular insult. Acta Medica Medianae. 2011; 50(2):511.

29. Kamberi B, Vasilevska K. Epidemiological study of the morbidity from first ever stroke in the Lower Pollog during 1990-2000. Medicus. 2005;3(1):37-41.

30. Kamberi B, Kamberi F. Proportions of stroke subtypes among hospitalized patients with first ever stroke in department of neurology and psychiatry in Tetovo (20052007). Medicus. 2008;10(2):131-136.

31. Kamberi B, Vasilevska K, Kamberi F. Contrasting presentation of the concentration of the homocysteine in the patients diagnosed with acute first ever ischaemic stroke, idiopathic Parkinson's disease and multiple sclerosis at the time of hospitalization. Medicus. 2011;15(1):68-75. 